\title{
Design of embedded intelligent home control system based on ZigBee wireless sensor networks
}

\author{
HaiLiu XIAO \\ Nanchang Institute of Science and Technology, Nanchang,330108, China \\ 346591653@163.com
}

Keywords: Smart home, ZigBee, data processing, Windows sockets

\begin{abstract}
Home wireless monitoring problem is the subject of today's cutting-edge research in the field of International Intelligent Building. Advent of wireless sensor networks to overcome the household wiring cumbersome, fully embodies the flexibility of smart home system, convenient and efficient. The project was developed based on research smart home monitoring system ZigBee technology and hitemet technology, remote monitoring and control of ZigBee hitemet short distance combined to achieve wireless home control and data acquisition systems, avoiding cabling, scalability. Firstly, the overall design of the system combined with the underlying ZigBee wireless sensor network characteristics and overall network monitoring system requirements, the system design is divided into four parts: the wireless transmission module, data processing module, an Ethernet transmission module, the PC display interface then ZigBee protocol standards to do a comprehensive research and analysis, and gives the CC2430-based wireless transmission module hardware and software design and star network structures and gives the test results.
\end{abstract}

\section{Introduction}

Compared with ordinary home, smart home features not only traditional residence features to provide comfort and safety, high quality and pleasant living space, also the original passive static structure into a dynamic tool has wisdom to help families keep the information with the outside exchange flow, optimize people's lifestyles, and even save money important feature intelligent building is for a variety of energy expenditure: networking, intelligent, humane, personal acceptance of intelligent products into their work and life, no longer for curiosity, but that demand intelligent system broadband integrated wiring system to bring people not only products, but more importantly, it embodies a service model and service concept [1-3]. The basic goal is to families with a variety of information related communications equipment, home appliances and home security devices, through a wired or wireless connection to a home intelligent system for centralized or remote monitoring, control and management of family things, keep these facilities and residential environment family harmony and coordination based on these outstanding advantages, housing information, intelligence will be an inevitable trend $[4,5]$.

From a technical perspective, smart home is the integration of computers, networks, automatic control, sensor and other technologies, and involving ecology, environment, energy and other fields of comprehensive systematic project, which is to achieve the final analysis, policy-based automatic control and exchange of information, so the smart home system is an integrated information flow and control flow of the network system. Progress is inseparable from the development of smart home related technology leap related technologies are bound to change the face of the smart home [6, 7].

Since the smart home is a multi-industry cross-coverage system engineering, various equipment manufacturers according to different interface standards and protocols of production equipment, the result is: the interconnection between different devices, interoperability becomes very difficult this problem is actually. By the communication protocol is no uniform standard household equipment caused in the smart home control systems, smart home is a home network to achieve basic communication inside the home of the nervous system, and the communication protocol is its essence, 
so the smart home design of the system, using the communication protocol with good development prospects is significant.

\section{ZigBee short-range wireless communications technology}

Although ZigBee communication protocol is for low-power and low-cost communications technology, but ZigBee protocol is to ensure the reliability of the communication, and the other big advantage ZigBee protocol is to make ad-hoc network, also very healing powers strong [8, 9].

1) Self-organizing capabilities of ZigBee: without human intervention, a network node can sense the presence of other nodes, and determine the connection between the composition of the network structure.

2) ZigBee self-healing function: add or remove a node, node locations are changed, node failure, etc., can be self-healing networks, and network topology accordingly adjusted, without human intervention, to ensure that the entire system can still normal work.

ZigBee protocol structure model was shown in Figure 1, can be seen from Figure 1, ZigBee Alliance provides a network layer, application layer design framework (APL), in which the frame of the application layer include application support layer (APS) or application programming interface, ZigBee device objects and application objects developed by the manufacturer. the network layer is responsible for determining the topology and maintenance, as well as naming and binding services, and complete addressing, routing, and security tasks. IEEE802.15.4 standard on the establishment of the network layer is designed here to self-organization and self-maintenance form.

\begin{tabular}{|c|c|c|}
\hline \multicolumn{2}{|c|}{ ZigBee application layer } & \multirow{5}{*}{ 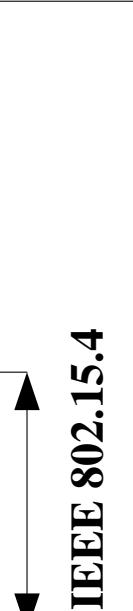 } \\
\hline ZigBee application & gram interface & \\
\hline Network layer(star-sh & d/nets/arborescence) & \\
\hline \multicolumn{2}{|c|}{ IEEE 802.15.4 MAC } & \\
\hline $\begin{array}{c}\text { IEEE 802.15.4 } \\
\text { 868/915 MHz PHY }\end{array}$ & $\begin{array}{c}\text { IEEE 802.15.4 } \\
2.4 \mathrm{GHz} \text { PHY }\end{array}$ & \\
\hline
\end{tabular}

Figure 1. ZigBee protocol structure model

\section{Home monitoring system}

\section{Hardware design}

Hardware design ZigBee node module is divided into portions and control communication radio interface section was shown in Figure 2. 


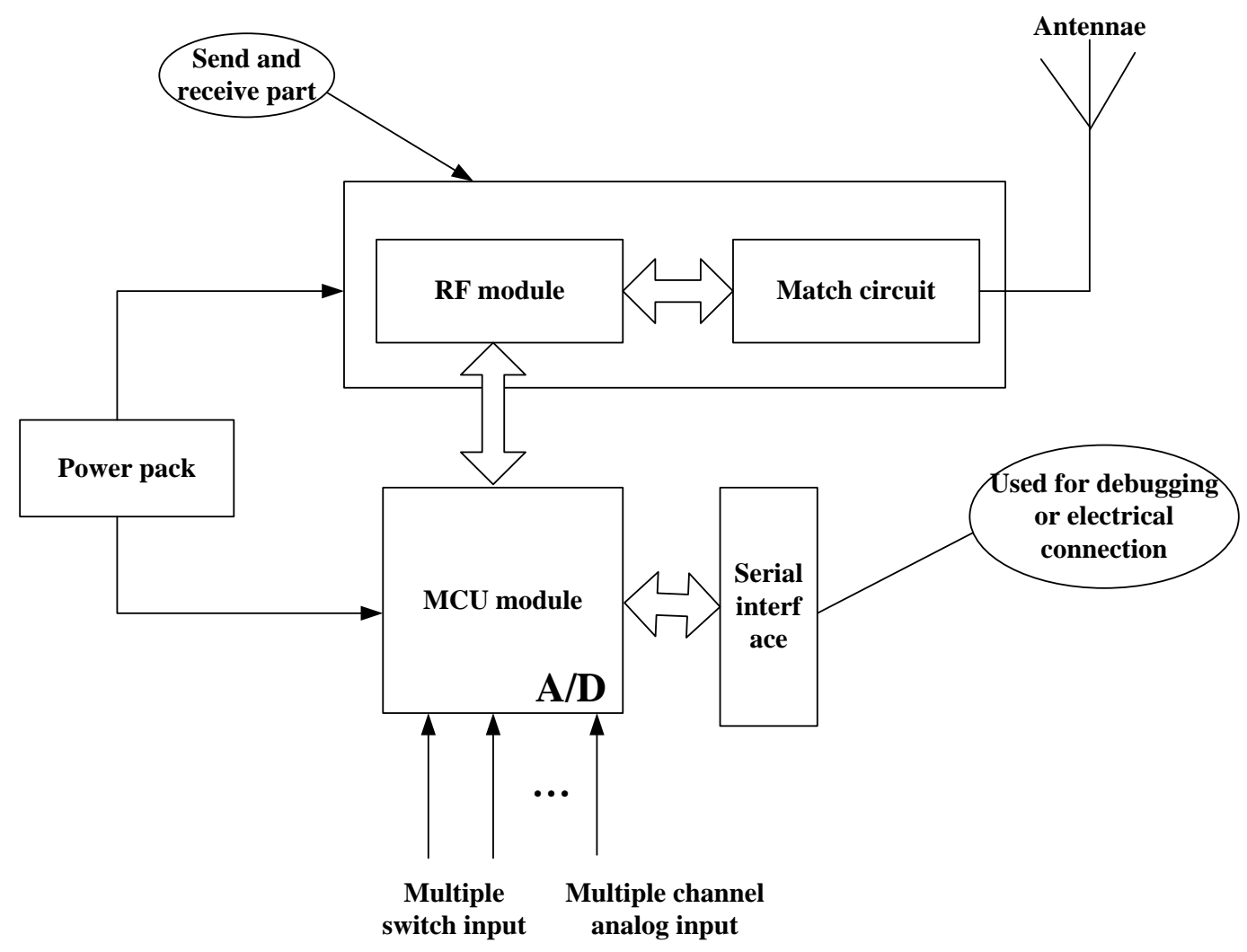

Figure 2. Hardware design ZigBee node module

Radio data communication part mainly responsible for inter-node; node from the serial interface can be connected to a PC in order to facilitate debugging or related electrical equipment can be connected to the interface, control electrical switches; and although the design of the master serial interface, but the main control communication interface is SPI, the data processing module for master chip TMS0F2812 exchange data transmission.

Any antenna is a radio communication system are an important and indispensable part of the antenna reasonably carefully chosen, we can achieve far distance communication and good communication effect. CC2430 and CC2420 antenna is the same, you can use two designs: metal PCB trace inverted-F antenna and a monopole antenna, PCB antenna lead is printed on the circuit board conductors, through which the air induction radio wave, a received signal. This design does not use the program, if you want to use this program, you can see CC2420 chip data sheet. Monopole antenna is a linear conductor is installed on the reflecting plane (the floor) by directly perpendicular (usually a length of not more than $1 / 4$ of the wavelength) composed of an antenna, its roots of the feeder. This design uses a monopole antenna 500, and the length of the antenna is $1 / 4$ of the wavelength of electromagnetic waves.

\section{Smart home system function analysis}

Smart home system mainly completes centralized management of home devices and control, improve safety, convenience, efficiency and comfort, smart home user home divided into two kinds of ordinary users and system administrators. Ordinary users of the user's system, the general needs of the system provides real-time temperature and humidity family management, lighting management systems, intelligent control and remote control home appliances, security monitoring, and other functions. System Administrator is a system management and maintenance, he security system safe and reliable operation, mainly to complete the user management, GSM information management, ZigBee network configuration work. The overall system functional block diagram was shown in Figure 3. 


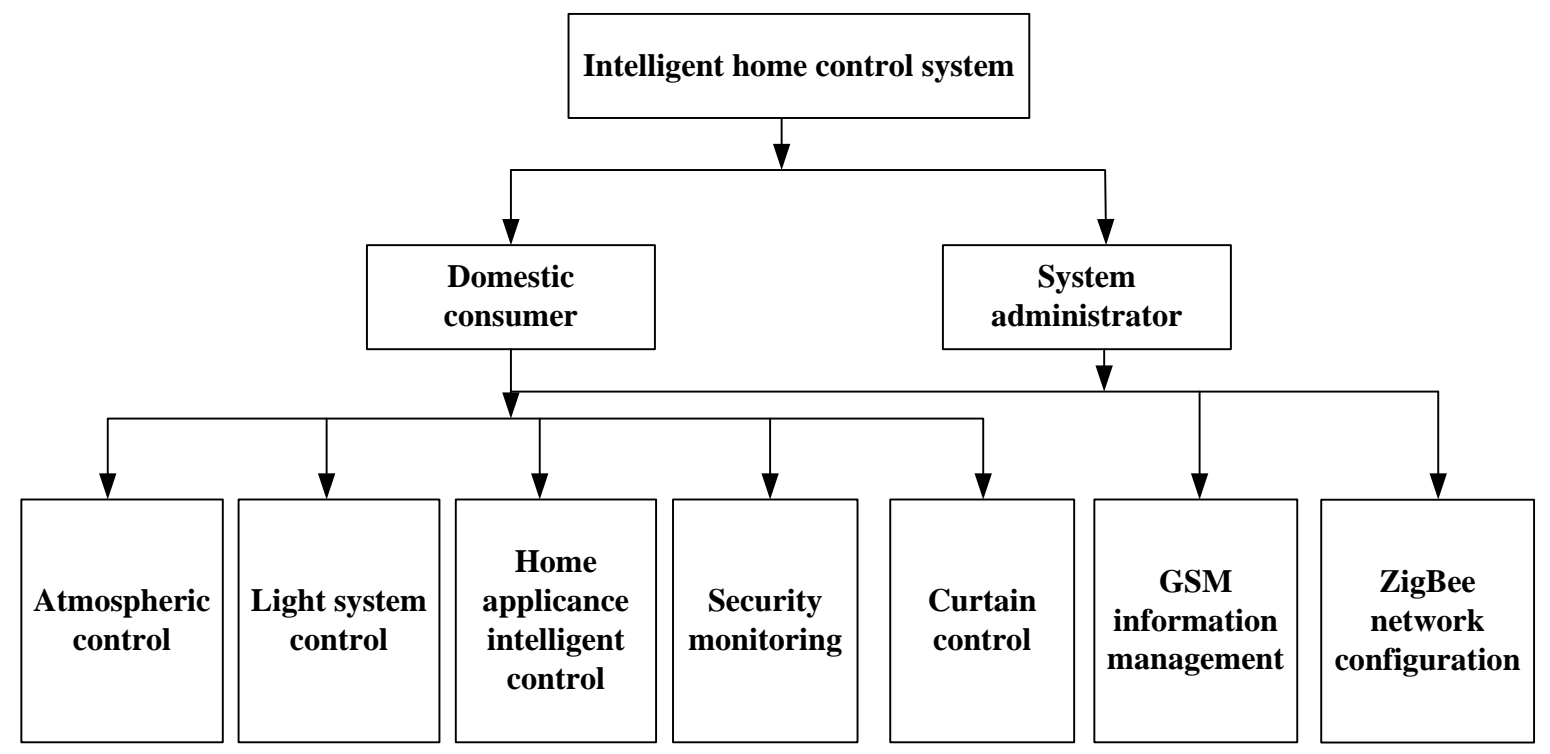

Figure 3. Functional block diagram of the overall system

Smart home system using micro-controller on the home device management and control, in order to improve scalability and reduce cable costs, the various devices connected to the system through home ZigBee wireless technology, and allows remote monitoring equipment through the GSM network.

\section{Summary}

The project was developed based on research smart home monitoring system ZigBee technology and hitemet technology, remote monitoring and control of ZigBee hitemet short distance combined to achieve wireless home control and data acquisition systems, avoiding cabling, scalability. Firstly, the overall design of the system combined with the underlying ZigBee wireless sensor network characteristics and overall network monitoring system requirements, the system design is divided into four parts: the wireless transmission module, data processing module, an Ethernet transmission module, the PC display interface then ZigBee protocol standards to do a comprehensive research and analysis, and gives the CC2430-based wireless transmission module hardware and software design and star network structures and gives the test results.

\section{References}

[1] Q. H. Spencer, A. L. Swindlehurst, M. Haardt. “Zero-Forcing Methods for Downlink Spatial Multiplexing in Multiuser MIMO Channel”, IEEE Transactions on Signal Processing, vol.52, no. 2, pp.461-471, May 2004.

[2] M. Sadek, A. Tarighat, A. H. Sayed, “A Leakage-based Precoding Scheme for Downlink multi-user MIMO Channels”, IEEE Transactions on Wireless Communications, vol. 26, no.8, pp.1505-1515, 2008.

[3] A. Tarighat, M. Sadek, A. H. Sayed, “A multi User Beamforming Scheme for Downlink MIMO Channels based on Maximizing Signal-to-Leakage Ratios”, IEEE International Conference on Acoustics, Speech, and Signal Processing, pp. 1129-1132, 2005.

[4] J.van de Beek,O. Edfors, M. Sandell, S. Wilson, P. Borjesson, “On Channel Estimation in OFDM System”, in Proceedings of the IEEE Vehicular Technology Conference, pp. 815-819, 1995.

[5] K.Wong, R. Cheng, K. B. Letaeif, R. D. Murch, "Adaptive antennas at the mobile and base stations in an OFDM/TDMA system”, IEEE Transactions on Communications, vol. 49, no.1, pp. 195-206, 2001. 
[6] M. Sadek, A. Tarighat, A. H. Sayed, "Active Antenna Selection in multi-user MIMO Communications,” IEEE Transactions on Signal Processing, vol. 55, no. 4, pp. 1498-1510, 2007.

[7] Zhu Yazhou, Zheng Guoxin, Rui Yun, , Li Mingqi, "A Novel Distributed Precoding Scheme Based on THP for Downlink Multi-Cell Multi-User OFDMA Wireless Systems", IJACT: International Journal of Advancements in Computing Technology, vol. 5, no. 9, pp. 213-220, 2011.

[8] Raore Soungalo, Li Renfa and Zeng Fanzi, "Evaluating and Improving Wireless Local Area Networks Performance", IJACT: International Journal of Advancements in Computing Technology, vol. 3, no. 2, pp. 156-164, 2011.

[9] L Tong, G Xu, B Hassibi, and T Kailath. Blind channel estimation based on second-order statistics: a frequency-domain approach. IEEE Trans. Inform. Theory, Vol. 41. Jan 1995. pp:329-334. 